\title{
Success rate in preterm uterine contraction inhibition with tocolytic agents in a tertiary care center
}

\section{Duangsamorn Kiatsuda Jadsada Thinkhamrop Witoon Prasertcharoensuk \\ Department of Obstetrics and Gynecology, Faculty of Medicine, Khon Kaen University, Khon Kaen, Thailand}

Correspondence: Jadsada Thinkhamrop Department of Obstetrics and Gynecology, Faculty of Medicine, Khon Kaen University, 123 Mittraphap Highway, Muang Khon Kaen, Khon Kaen 40002, Thailand

Tel +66 43202489

Fax +66 43348395

Email jadsada@kku.ac.th
This article was published in the following Dove Press journal:

International Journal of Women's Health

15 November 2016

Number of times this article has been viewed

Objective: This study aims to assess the success rate of inhibiting preterm uterine contraction with tocolytic agents to delay delivery for at least 48 hours and risk factors of failure inhibition.

Materials and methods: Between January 2013 and July 2014, medical records of all singleton pregnant women between $24{ }^{0 / 7}$ and $33^{6 / 7}$ weeks of gestation with the diagnosis of preterm labor (with cervical dilatation) or threatened preterm labor (without cervical dilatation) who received tocolytic agents were reviewed. The success rate of preterm uterine contraction inhibition was accounted in patients with 48 hours delayed delivery. The risk factors of the inhibition failure and neonatal outcomes were also investigated in this study.

Results: Among 424 pregnant women diagnosed of preterm labor or threatened preterm labor, 103 singleton pregnant women met the study criteria. Overall success rate of preterm uterine contraction inhibition to prolong pregnancy for at least 48 hours was $86.4 \%$ ( $95 \%$ confidence interval $[\mathrm{CI}]: 78.3,92.3)$. However, the success rate among the threatened preterm labor group was $93.8 \%$ (95\% CI: 88.3, 99.1) while the preterm labor group was $60.9 \%(95 \%$ CI: $39.3,82.4)$. The significant factor associated with inhibition failure was preterm labor (adjusted odds ratio 7.22; 95\% CI: 1.99, 26.20).

Conclusion: The success rate of preterm uterine contraction inhibition with tocolytic agents to delay delivery for at least 48 hours was high in threatened preterm labor and low in preterm labor. A significant risk factor for inhibition failure was the preterm uterine contraction with cervical change.

Keywords: inhibition of uterine contraction, success rate, preterm labor, threatened preterm labor, tocolytic agents

\section{Introduction}

Preterm delivery is the main leading cause of perinatal morbidity and mortality. Furthermore, it is the most common cause of antenatal hospital admission. ${ }^{1}$ The incidence of preterm birth rate in US was $12 \%$ in $2011 .^{2,3}$ At Siriraj Hospital, a tertiary care in central part of Thailand, the preterm birth rate was 9.4\% in 2004 and $13.7 \%$ in 2010. ${ }^{4}$ Srinagarind Hospital is another tertiary care center in northeastern part of Thailand; the preterm birth rate was as high as 11.5\% in 2013 (unpublished data).

The complications of preterm births arise from immature organ systems that are not yet ready to support life in the extrauterine environment. For instance, respiratory distress syndrome is caused by a lack of surfactants in the lung epithelium. Antenatal administration of corticosteroids to women who were expected to give preterm birth was associated with a significant reduction of neonatal morbidity and mortality, respiratory distress syndrome, intraventricular hemorrhage, and necrotizing 
enterocolitis of preterm infants. National Institute of Health and the American College of Obstetrician and Gynecologists recommended a single course of corticosteroids for pregnant women between 24 and 34 weeks of gestation who are at risk of preterm delivery within 7 days. ${ }^{5,6}$ The use of tocolytic agents to suppress preterm uterine contraction can delay delivery for at least 48 hours, and this prolongation enable the complete administration of corticosteroids to obtain their maximum effect for gestational age of 24-34 weeks. ${ }^{6}$

Despite of the available modalities to prevent preterm birth and its consequences, the proportions of preterm birth are not reduced. ${ }^{7}$ Therefore, we planned to evaluate the success rate of preterm uterine contraction inhibition with tocolytic agents and factors associated with inhibition failure to aware the possibility of preterm delivery.

\section{Materials and methods}

This observational retrospective study was approved by the Khon Kaen University Ethics Committee for Human Research based on the Declaration of Helsinki and the ICH Good Clinical Practice Guidelines (HE571337). After reviewing the data collection forms from the medical records of the project, the Ethics Committee deemed patient written informed consent was not required, as this was a retrospective study. We have reviewed the medical records of all singleton pregnant women between $24^{0 / 7}$ and $33^{6 / 7}$ weeks of gestation admitted to the labor room of Srinagarind Hospital, Khon Kaen University, Thailand, between January 2013 and July 2014, and selected the patients who received uterine contraction inhibition with tocolytic drugs under diagnosis of preterm labor or threatened preterm labor. Multiple pregnancies, women with diagnosis of dead fetus in utero, and lethal fetal anomalies were excluded. Preterm labor was defined as regular uterine contractions accompanied by a change in cervical dilatation, effacement, or both. ${ }^{6}$ Threatened preterm labor was defined as regular uterine contractions occurring at the frequency of at least once in every 10 minutes during 30 minutes of monitoring, with no dilatation and effacement of cervix. ${ }^{4}$ The high risk of preterm delivery was defined as group of women with any history of obstetric, medical, or surgical complication during pregnancy. The gestational age determination was based on the certain date of the last menstrual period or first trimester ultrasound examination.

Their medical records were assessed for the baseline characteristics including age, weight, gravidity, parity, gestational age on admission, numbers of antenatal care visit, characteristics of uterine contraction, and cervical status to classify studied women to preterm labor or threatened preterm labor. The types, modes, duration of administration, and the adverse effects of the tocolytic agents used for uterine contraction inhibition were also retrieved. The pregnancy prolongation was measured in terms of the duration (days) and the gestational age (complete weeks) whether it was beyond 34 and 37 weeks or not. Additionally, we also reviewed all of neonatal outcomes including gestational age, route of delivery, appearance, pulse, grimace, activity, respiration (APGAR) scores, and birth weight.

The tocolytic agents commonly used in Srinagarind Hospital are Adalat ${ }^{\circledR}$ (nifedipine), Bricanyl ${ }^{\circledR}$ (terbutaline), and Ventolin ${ }^{\circledR}$ (salbutamol). The course of oral nifedipine (10 mg) started with the initial load every 15 minutes with the maximum dose of $40 \mathrm{mg}$ until no uterine contraction was observed, followed by the maintenance dose of $10 \mathrm{mg}$ every 6 hours for 24 hours, an then tapered every 8 hours for 24 hours, every 12 hours for 24 hours, and once daily, and discontinue if there was no more contraction. For terbutaline, 4 ampules $(2 \mathrm{mg}$ ) were added to $5 \% \mathrm{D} / \mathrm{W} 100 \mathrm{~mL}$ and intravenously dripped beginning with the rate of 5 microdrops $/ \mathrm{min}$, then increased 5 microdrops/min every 30 minutes until there was no uterine contraction or getting the maximum rate of 45 microdrops/ min. If there was no uterine contraction for 24 hours, the dripping rate would be reduced to 5 microdrops/min until off. In case of salbutamol, 10-20 mg of salbutamol was orally given every 6 hours until uterine contraction disappeared. If the preterm pregnant women still have uterine contraction while using these drug regimens or have some intolerable side effects, they were classified as inhibition failure and the attending physicians would change to other drugs or none. Doses of these drugs could be modified in some cases, depending on the associated maternal or fetal conditions and the judgment of attending physicians.

Since in our setting, only preterm pregnant women of $24^{0 / 7}$ to $33^{6 / 7}$ weeks of gestation would receive a course of antenatal corticosteroid administration for accelerate fetal lung maturation. So the success of preterm uterine contraction inhibition was assessed as the delay in delivery for at least 48 hours after tocolytics administration between 24 and 33 week of gestation while the inhibition failure was defined as the delay in delivery for $<48$ hours. The risk factors of the inhibition failure were also analyzed in this study.

The sample size calculation based on the objective to assess the success rate of preterm uterine contraction inhibition with the maximal proportion of defined women was $0.5 \%$ and $10 \%$ of allowance error. The study required at least 96 singleton pregnant women who admitted to the labor room with preterm uterine contraction and received tocolytic agents for inhibition.

For statistical analysis, mean, standard deviation (SD), number, and percentage were calculated. The chi-square and 
Fisher's exact test were used to evaluate the risk factors of labor inhibition failure. These variables were then included if $P$-value $<0.20$ in a stepwise logistic regression analysis to determine which factors, if any, were independently associated with the risk of labor inhibition failure. Adjusted odds ratio with a $95 \%$ confidence interval $(\mathrm{CI})$, which did not include unity were considered statistically significant.

\section{Results}

During the studied period, a total of 424 pregnant women met the diagnosis of preterm labor or threatened preterm labor. After exclusion of 39 multiple pregnancies, a women with dead fetus in utero, 2 women with lethal fetal anomalies, 3 women whose medical records were lost, 262 women between $34^{0 / 7}$ and $36^{6 / 7}$ weeks of gestation and also 14 women between $24^{0 / 7}$ and $33{ }^{6 / 7}$ weeks of gestation whose uterine contractions were not inhibited, 103 singleton pregnant women at $24^{0 / 7}$ to $33^{6 / 7}$ weeks of gestation who received tocolytic agents were reviewed.

The baseline characteristics of the studied women were shown in Table 1. Their mean age was 28 years old. Around three-fourth of them $(77.7 \%)$ met the clinical criteria of threatened preterm labor, while $22.3 \%$ met the diagnosis of preterm labor. There were histories of obstetrical, medical, and/or surgical complications during pregnancy among studied women. Anemia was the most frequently associated condition (34\%), followed by previous abortion (29.1\%), preterm pre-labor rupture membranes $(17.5 \%)$, urinary tract infection or asymptomatic bacteriuria $(15.5 \%)$, and history of preterm labor/delivery (11.7\%) and vaginal bleeding (9.7\%). Few cases of other complications were autoimmune diseases ( 3 cases), genital tract infection ( 3 cases), infective diarrhea/food poisoning ( 3 cases), polyhydramnios ( 3 cases), and 1 case each of chorioamnionitis, sepsis, uterine leiomyoma, abdominal surgery during pregnancy, and uterine cervical surgery.

Table I Baseline characteristics of pregnant women

\begin{tabular}{ll}
\hline Characteristics & Mean \pm SD or N (\%) \\
\hline Age, years & $28.4 \pm 7.0$ \\
Prepregnancy BMl, $\mathrm{kg} / \mathrm{m}^{2}$ & $21.0 \pm 3.5$ \\
$\quad<18.5$ (underweight) & $23(22.3)$ \\
$\quad \geq 30$ (obesity) & $4(3.9)$ \\
Primiparity & $66(64.1)$ \\
Gestational age on admission, weeks & $30.8 \pm 2.4$ \\
Number of antenatal care visit & $7.0 \pm 2.7$ \\
History of pregnancy complications & $80(77.7)$ \\
Uterine contraction and cervical condition & \\
$\quad$ Threatened preterm labor & $80(77.7)$ \\
Preterm labor & $23(22.3)$ \\
\hline
\end{tabular}

Abbreviations: BMI, body mass index; SD, standard deviation.
In Table 2, the overall success rate of preterm uterine contraction inhibition for prolongation of pregnancy at least for 48 hours was $86.4 \%$. The success rate among who were diagnosed as threatened preterm labor on admission was high (93.8\%), while the success rate among who were diagnosed as preterm labor on admission was low (60.9\%). High risk of preterm delivery group had high success rate $(85.0 \%)$. The more cervical dilatation group had the more failure rate. Calcium channel blocker had the better effect than betamimetics and combination of tocolytics. More than $80 \%$ of included women received complete course of corticosteroid before delivery. Approximately half of the studied women (55.3\%) had prolonged pregnancy beyond 34 weeks. One-third of them $(38.8 \%)$ gave birth after 37 weeks of gestation.

Regarding the type of tocolytic agents, terbutaline was the most frequently selected tocolytic agent, followed by nifedipine. Intolerable side effects of tocolytic agents were observed in 13 cases, whose medication was discontinued. Among these 13 cases, 5 cases developed hypotension after nifedipine treatment and 8 cases showed maternal tachycardia/dyspnea and fetal tachycardia after terbutaline treatment. Duration of tocolytic agent administration for labor inhibition was $2-5$ days (median $=4$ days). Pregnancy could be prolonged for 4-42 days (median $=21$ days).

The neonatal outcomes were shown in Table 3. Mean baby birth weight was 2,370.9 $\mathrm{g}$ (SD: 833.6), and their mean gestational age at birth was 34.4 weeks (SD: 3.5 ). About twothird $(61.2 \%)$ of them were born before 37 weeks. Almost half of them $(49.5 \%)$ were low birth weight $(<2,500 \mathrm{~g})$ babies. Cesarean section was the majority route of delivery. There were 4 cases of severe birth asphyxia whose APGAR score was $<4$ at 5 minutes after birth.

Table 2 Success rate of preterm uterine contraction inhibition

\begin{tabular}{ll}
\hline Group of studied women & Success rate $(95 \% \mathbf{C l})$ \\
\hline All uterine contraction inhibition & $86.4(78.3,92.3)$ \\
Threatened preterm labor $(\mathrm{n}=80)$ & $93.8(88.3,99.1)$ \\
Preterm labor $(\mathrm{n}=23)$ & $60.9(39.3,82.4)$ \\
High risk of preterm delivery $(\mathrm{n}=80)$ & $85.0(77.0,93.0)$ \\
Cervical dilatation, cm & \\
I $(\mathrm{n}=8)$ & $75.0(36.3,100)$ \\
$2-3$ ( $\mathrm{n}=\mathrm{II})$ & $54.5(36.3,100)$ \\
$\geq 4(\mathrm{n}=4)$ & $50.0(0,100)$ \\
Type of tocolytics & \\
CCB ( $\mathrm{n}=28)$ & $96.4(89.1,100)$ \\
Betamimetics $(\mathrm{n}=45)$ & $86.7(76.3,96.9)$ \\
Combine CCB + betamimetics $(\mathrm{n}=30)$ & $76.7(60.6,92.7)$ \\
Completed course of corticosteroids & $85.4(77.1,91.6)$ \\
Delivery after 34 weeks gestational age & $55.3(45.6,65.1)$ \\
Delivery after 37 weeks gestational age & $38.8(29.3,48.4)$ \\
\hline
\end{tabular}

Abbreviations: $\mathrm{CCB}$, calcium channel blocker; $\mathrm{Cl}$, confidence interval. 
Table 3 Neonatal outcomes

\begin{tabular}{ll}
\hline Neonatal outcomes & $\mathbf{N}(\%)$ \\
\hline Birth weight $<2,500 \mathrm{~g}$ & $5 \mathrm{I}(49.5)$ \\
Route of delivery & \\
$\quad$ Spontaneous vaginal delivery & $39(37.9)$ \\
Cesarean section & $60(58.3)$ \\
Vacuum and extraction & $2(1.9)$ \\
Breech assisting & $2(1.9)$ \\
APGAR score & \\
At the 5th minute $<6$ & $9(8.7)$ \\
At the 5th minute $<4$ & $4(3.9)$ \\
\hline
\end{tabular}

Abbreviation: APGAR, appearance, pulse, grimace, activity, respiration.

In the univariate analysis, risk factors including preeclampsia with severe feature, history of abortion, more than 24 hours of uterine contraction, and history of cervical surgery were not significantly associated with inhibition failure ( $P$-value $>0.20)$. When using multivariate logistic regression analysis, only preterm labor had statistical significant associated with inhibition failure. The results are given in Table 4.

\section{Discussion}

Premature delivery is a common complication of pregnancy. Estimated worldwide incidence of preterm birth was 9.6\% in 2005, mostly occurred in Africa and Asia. ${ }^{8}$ In 2010, low birth weight babies worldwide were estimated to be around 18 million, and $59 \%$ of them were small-for-gestational age term and $41 \%$ were preterm. Two-third of small-forgestational age infants were born in Asia. ${ }^{9}$ The understanding of the causes of premature delivery, low birth weight babies and improvement of the effectiveness of available modality of intervention are needed to improve birth outcomes. The results of this study from a tertiary care hospital in northeastern Thailand showed the same tendency of worldwide burden of preterm pregnancy complications. We found $49.5 \%$ of newborns were low birth weight. In the present study, women with uterine contraction before 34 weeks of gestation were admitted to the hospital to prolong pregnancy with tocolytic agents. The majority of them (86.4\%) could continue pregnancy at least 48 hours after receiving the tocolytic agents and receive completed corticosteroids course to accelerate fetal lung maturity. However, $77.7 \%$ of these studied women presented with threatened preterm labor, which is defined as uterine contraction without cervical dilatation. The significant risk factor of inhibition failure was preterm uterine contraction with cervical dilatation (preterm labor).

Similar to the authors' present results, Motazedian et al reported that a 48-hour prolongation of pregnancy was achieved in $87 \%$ patients receiving terbutaline and $84 \%$ patients receiving salbutamol. ${ }^{10}$ Likewise, the study at Siriraj Hospital, Bangkok, Thailand, revealed that the success rate in threatened preterm labor inhibition for 12 hours with nifedipine was about $80 \% .{ }^{11}$ However, in cases of women with advanced cervical dilatation (4-8 cm) who received one or more tocolytics (magnesium sulfate, indomethacin, or nifedipine), only $23 \%$ could prolong pregnancy at least 48 hours after admission. ${ }^{12}$ Similarly, in cases of women with the diagnosis of preterm labor based on the presence of uterine contraction with documented cervical change, success rate by nifedipine was $70 \% .{ }^{13}$ In these two studies with the lower success rates, the patients presented as preterm labor with apparent cervical changes. We noticed that, regardless of number and the type of tocolysis, the success rate was higher when uterine contraction inhibition was applied to women with preterm uterine contraction without cervical changes. In the present study, the success rate to prolong pregnancy at least 48 hours was $93.8 \%$ in threatened preterm labor group and that of preterm labor group was $60.9 \%$. Furthermore, the authors' data revealed that preterm labor (uterine contraction with cervical change) was the significant risk factor of labor inhibition failure.

The main rationale of tocolysis for preterm labor are to delay delivery for at least 48 hours to allow completed course of corticosteroids to accelerate fetal lung surfactant production and allow to transfer mother to a tertiary facility. Betamimetics and calcium channel blockers reduce the number of preterm labor women who will give birth within 48 hours. Nevertheless, tocolysis does not appear to significantly lengthen the gestational age beyond 7 days. ${ }^{14}$ It is known that nifedipine is easy to administer and have fewer side effects relative to betamimetics. ${ }^{15}$ The authors' results also support this notion as intolerable side effects occurred more in cases of betamimetics treatment.

Table 4 Risk factors of inhibition failure

\begin{tabular}{lllll}
\hline Risk factors & $\begin{array}{l}\text { Inhibition failure } \\
(\mathbf{N}=\mathbf{1 4})\end{array}$ & $\begin{array}{l}\text { Inhibition success } \\
\mathbf{( N = 8 9 )}\end{array}$ & $\begin{array}{l}\text { Univariate } \\
\text { P-value }\end{array}$ & Adjusted OR (95\% Cl) \\
\hline Preterm labor* & 9 & 14 & $<0.00 \mathrm{I}$ & $7.22(\mathrm{I.99,26.20)}$ \\
Intolerable side effects & 4 & 9 & 0.065 & $2.43(0.52,11.33)$ \\
PPROM & 5 & 13 & 0.063 & $1.99(0.48,8.33)$ \\
Maternal BMI $<18.5 \mathrm{~kg} / \mathrm{m}^{2}$ & 6 & 17 & 0.056 & $2.63(0.69,10.09)$ \\
\hline
\end{tabular}

Note: *Regular uterine contractions accompanied by a change in cervical dilatation, effacement, or both.

Abbreviations: $\mathrm{BMI}$, body mass index; $\mathrm{Cl}$, confidence interval; OR, odds ratio; PPROM, preterm pre-labor rupture membranes. 
In spite of the success rate of preterm uterine contraction inhibition was as high as $86.4 \%$ in this study, there were substantial perinatal complications associated with premature delivery. For example, the mean birth weight of babies was approximately 2,300 $\mathrm{g}$ and a half of them had low birth weight $(<2,500 \mathrm{~g})$. Severe birth asphyxia was seen in 3.9\% and more than half $(58.3 \%)$ of the delivery required surgical intervention. Thus, we should provide advanced neonatal care and prepare for complicated delivery for the pregnancy complicated with preterm uterine contraction with or without cervical dilatation.

Limitations of this study were those inherent to any retrospective study which made it difficult to gain the complete data. Fortunately, most of the outcomes of interest were available in the retrieved medical records. Another was the limited number of studied women, in that the majority of women enrolled in this study were those with threatened preterm labor that would lead to overestimation of the overall success rate of uterine contraction inhibition. However, we tried to minimize such effects by separating the patients into threatened preterm and preterm labor groups for outcome analysis.

Regarding the evaluation of pregnancy outcomes of uterine contraction inhibition with tocolytic agents, further research on threatened preterm labor with or without tocolysis would be necessary. Also, whether preterm uterine contraction with cervical change should get high success rate as preterm uterine contraction without cervical change if the tocolytics could attenuate uterine contraction should be explored in future.

\section{Conclusion}

The success rate of preterm uterine contraction inhibition with tocolytic agents to delay delivery for at least 48 hours was high in threatened preterm labor and low in preterm labor. A significant risk factor for inhibition failure was the preterm uterine contraction with cervical change.

\section{Acknowledgments}

The authors wish to thank the Faculty of Medicine for the financial support (grant number IN58103). We also would like to present our appreciation for the technical support of data analysis from Clinical Epidemiology Unit, Faculty of Medicine and Yukifumi Nawa for editing the English of the manuscript via Publication Clinic, Khon Kaen University, Thailand.

\section{Disclosure}

The authors report no conflicts of interest in this work.

\section{References}

1. Tucker JM, Goldenberg RL, Davis RO, Copper RL, Winkler CL, Hauth JC. Etiologies of preterm birth in an indigent population: is prevention a logical expectation? Obstet Gynecol. 1991;77(3):343-347.

2. Martin JA, Osterman MJ, Sutton PD. Are preterm births on the decline in the United States? Recent data from the National Vital Statistics System. NCHS Data Brief. 2010;39:1-8.

3. Goldenberg RL, Culhane JF, Iams JD, Romero R. Epidemiology and causes of preterm birth. Lancet. 2008;371(9606):75-84.

4. Chawanpaiboon S, Kanokpongsakdi S. Preterm birth at Siriraj Hospital: a 9-year period review (2002-2010). Siriraj Med J. 2011 63(5):143-146.

5. Antenatal corticosteroids revisited: repeat courses - National Institutes of Health Consensus Development Conference Statement, August 17-18, 2000. Obstet Gynecol. 2001;98(1):144-150.

6. ACOG practice bulletin no. 127: management of preterm labor. Obstet Gynecol. 2012;119(6):1308-1317.

7. Norwitz ER, Caughey AB. Progesterone supplementation and the prevention of preterm birth. Rev Obstet Gynecol. 2011;4(2):60-72.

8. Beck S, Wojdyla D, Say L, et al. The worldwide incidence of preterm birth: a systematic review of maternal mortality and morbidity. Bull World Health Organ. 2010;88(1):31-38.

9. Lee AC, Katz J, Blencowe H, et al. National and regional estimates of term and preterm babies born small for gestational age in 138 low-income and middle-income countries in 2010. Lancet Glob Health. 2013;1(1): e26-e36.

10. Motazedian S, Ghaffarpasand F, Mojtahedi K, Asadi N. Terbutaline versus salbutamol for suppression of preterm labor: a randomized clinical trial. Ann Saudi Med. 2010;30(5):370-375.

11. Chawanpaiboon S, Pimol K, Sirisomboon R. Comparison of success rate of nifedipine, progesterone, and bed rest for inhibiting uterine contraction in threatened preterm labor. J Obstet Gynaecol Res. 2011;37(7):787-791.

12. Manuck TA, Herrera CA, Korgenski EK, et al. Tocolysis for women with early spontaneous preterm labor and advanced cervical dilation. Obstet Gynecol. 2015;126(5):954-961.

13. Nikbakht R, Taheri Moghadam M, Ghane'ee H. Nifedipine compared to magnesium sulfate for treating preterm labor: a randomized clinical trial. Iran J Reprod Med. 2014;12(2):145-150.

14. Haram K, Mortensen JH, Morrison JC. Tocolysis for acute preterm labor: does anything work. J Matern Fetal Neonatal Med. 2015;28(4): 371-378.

15. Gaspar R, Hajagos-Toth J. Calcium channel blockers as tocolytics: principles of their actions, adverse effects and therapeutic combinations. Pharmaceuticals (Basel). 2013;6(6):689-699.
International Journal of Women's Health

\section{Publish your work in this journal}

The International Journal of Women's Health is an international, peerreviewed open-access journal publishing original research, reports, editorials, reviews and commentaries on all aspects of women's healthcare including gynecology, obstetrics, and breast cancer. The manuscript management system is completely online and includes

\section{Dovepress}

a very quick and fair peer-review system, which is all easy to use. Visit http://www.dovepress.com/testimonials.php to read real quotes from published authors. 